\title{
JACOBI POLYNOMIAL EXPANSIONS WITH POSITIVE COEFFICIENTS AND IMBEDDINGS OF PROJECTIVE SPACES
}

\author{
BY RICHARD ASKEY ${ }^{1}$
}

To I. J. Schoenberg on his 65 th birthday

Communicated by H. Helson, September 25, 1967

In much of Schoenberg's work there has been a strong interconnection between analytic and geometric reasoning. Here we use a remark he made about imbeddings of metric spaces to prove part of a conjecture about when a Jacobi polynomial $P_{n}^{(\gamma, \delta)}(x)$ can be expanded in terms of another $P_{k}^{(\alpha, \beta)}(x)$ with nonnegative coefficients. Also we get from a different special case of this conjecture some nonimbedding theorems for projective spaces.

$P_{n}^{(\alpha, \beta)}(x)$, the Jacobi polynomial of degree $n$, order $(\alpha, \beta), \alpha, \beta>-1$, is defined by

(1) $(1-x)^{\alpha}(1+x)^{\beta} P_{n}^{(\alpha, \beta)}(x)=\frac{(-1)^{n}}{2^{n} n !}\left(\frac{d}{d x}\right)^{n}\left[(1-x)^{n+\alpha}(1+x)^{n+\beta}\right]$.

These polynomials are orthogonal on $(-1,1)$ with respect to the weight function $(1-x)^{\alpha}(1+x)^{\beta}$ and what is crucial for us is that $P_{n}^{(\alpha, \beta)}(1)>0$. We consider the expansion

$$
P_{n}^{(\gamma, \delta)}(x)=\sum_{k=0}^{n} \alpha_{k} P_{k}^{(\alpha, \beta)}(x)
$$

and ask for what values of $\alpha, \beta, \gamma, \delta$ are all the coefficients $\alpha_{k}, k=0$, $1, \cdots, n$, nonnegative. For $\beta=\delta$ and $\gamma>\alpha$ the $\alpha_{k}$ were computed by Szegö [8] and were found to be positive. He used this relation to solve the end point Cesàro summability problem for Jacobi series.

For $\alpha=\beta, \gamma=\delta$ the $\alpha_{k}$ were given by Gegenbauer [5] and again they are nonnegative for $\alpha>\gamma$. This has been used by Hua [6] and Askey and Wainger [1]. Actually this result of Gegenbauer is a special case of Szegö's result. For

$$
\frac{P_{n}^{(\alpha,-1 / 2)}\left(2 x^{2}-1\right)}{P_{n}^{(\alpha,-1 / 2)}(1)}=\frac{P_{2 n}^{(\alpha, \alpha)}(x)}{P_{2 n}^{(\alpha, \alpha)}(1)}
$$

${ }^{1}$ Supported in part by N.S.F. grant GP-6764. 
and

$$
\frac{x P_{n}^{(\alpha, 1 / 2)}\left(2 x^{2}-1\right)}{P_{n}^{(\alpha, 1 / 2)}(1)}=\frac{P_{2 n+1}^{(\alpha, \alpha)}(x)}{P_{2 n+1}^{(\alpha, \alpha)}(1)} .
$$

Thus (2) for $\beta=\delta=-\frac{1}{2}$ is equivalent to (2) for $n$ even, $\alpha=\beta, \gamma=\delta$; and (2) for $\beta=\delta=\frac{1}{2}$ is equivalent to (2) for $n$ odd, $\alpha=\beta, \gamma=\delta$. Since the proof of Szegö's result is easier and more natural than any proof I know of Gegenbauer's result, I like to think of Szegö's result as the more fundamental. However, it would be nice to have $\alpha_{k}$ in the general case (2) and to get the positivity for the known cases from the general case. Unfortunately I am unable to find a simple enough formula for $\alpha_{k}$. $\alpha_{k}$ has been computed by Feldheim [3] and he gets it as a ${ }_{3} F_{2}$. I haven't seen his proof, but a proof using (1) a couple of times, many integrations by parts and the binomial theorem is easy. This proof is identical with Szegö's proof for $\beta=\delta$ until the last step when $(1+x)^{c}$ is expanded in terms of $(1-x)^{j}$. Explicitly

$$
\begin{aligned}
a_{k}= & \frac{(2 k+\alpha+\beta+1) \Gamma(k+\alpha+\beta+1) \Gamma(n+k+\gamma+\delta+1) \Gamma(n+\delta+1) \Gamma(n-k+\gamma-\alpha)}{\Gamma(\gamma-\alpha) \Gamma(k+\beta+1) \Gamma(n+k+\alpha+\delta+1) \Gamma(n+\gamma+\delta+2) \Gamma(n-k+1)} \\
& \cdot{ }_{3} F_{2}(\delta-\beta, \alpha-\gamma+1, \alpha+k+1 ; \alpha-\gamma+k-n+1, n+k+\alpha+\delta+2 ; 1) .
\end{aligned}
$$

A reasonable conjecture which includes both of the above cases is that $\alpha_{k} \geqq 0$ if $(\gamma, \delta)$ lies in the triangular region above the line $\delta=\beta$ and to the right of the line through $(\alpha, \beta)$ and $(-1,-1)$. By Szegö's result it would be sufficient to show this for $(\gamma, \delta)$ on the line through $(-1,-1)$ and $(\alpha, \beta)$. This is one of a number of problems that is equivalent to a certain ${ }_{3} F_{2}$ being positive. It seems that a systematic study of when these and other generalized hypergeometric functions are positive would yield many interesting results.

This conjecture is false for $(\gamma, \delta)$ above the line through $(-1,-1)$ and $(\alpha, \beta) . P_{1}^{(\alpha, \beta)}(x)=\frac{1}{2}[(\alpha+\beta+2) x+(\alpha-\beta)]$ and $P_{0}^{(\alpha, \beta)}(x)=1$. A computation shows that

$$
\begin{aligned}
P_{1}^{(\gamma, \delta)}(x)= & \left(\frac{\gamma+\delta+2}{\alpha+\beta+2}\right) P_{1}^{(\alpha, \beta)}(x) \\
& +\frac{[(\gamma-\delta)(\alpha+\beta+2)+(\beta-\alpha)(\gamma+\delta+2)]}{2(\alpha+\beta+2)} P_{0}^{(\alpha, \beta)}(x)
\end{aligned}
$$

and the second coefficient is nonnegative if and only if $\gamma$ $\geqq((\alpha+1)(\delta+1) /(\beta+1))-1$, i.e. $(\gamma, \delta)$ lies to the right of the given line.

This remark has an interesting consequence when combined with 
some work on Bochner on positive definite functions on Riemannian spaces. Schoenberg defined a function $f$ on $[0, \infty]$ as positive definite on a separable metric space $X$ if $\sum_{i, j=0}^{n} f\left(\operatorname{dist}\left(x_{i}, x_{j}\right)\right) \rho_{i} \bar{\rho}_{j} \geqq 0$ for all $x_{i} \in X$ and complex $\rho_{i}$. For the sphere $S^{k}$ he has found all the positive definite functions [7] and they are just $f(\theta)=\sum_{n=0}^{\infty} a_{n} P_{n}^{(\alpha, \alpha)}(\cos \theta)$ with $\sum a_{n} P_{n}^{(\alpha, \alpha)}(1)<\infty, a_{n} \geqq 0$. Here $\alpha=(k-3) / 2$. Since $S_{k}$ can be isometrically imbedded in $S_{l}$ for $k<l$, it follows that $P_{n}^{(\gamma, \gamma)}(\cos \theta)$ $=\sum_{k=0}^{n} \alpha_{k} P_{k}^{(\alpha, \alpha)}(\cos \theta)$ with $\alpha_{k} \geqq 0$ for $\gamma>\alpha$ and $\gamma, \alpha$ half integers, as Schoenberg observed. This remark that the isometric imbedding of a metric space in a second metric space gives rise to a reverse inclusion in their positive definite functions can be used to obtain a couple of interesting results when combined with work of Bochner. For a number of Riemannian manifolds, including the real projective spaces $P^{d}(R)$, the complex projective spaces, $P^{d}(C)$, the quaternionic projective spaces $P^{d}(H)$, and the Cayley elliptic plane $P^{16}$, Bochner has found the positive definite functions [2]. Here $d$ is the real dimension of the space. They are $\sum_{n=0}^{\infty} a_{n} \phi_{n}$, with $a_{n} \geqq 0$ and $\phi_{n}$ the spherical function of degree $n$. These spherical functions are Jacobi polynomials. For $P^{d}(R)$ they are given in [4] as $P_{2 n}^{(\alpha, \alpha)}(\cos (\pi \theta / 2 L))$ where $L$ is the diameter of the space in question. Using (3) we see that they are also $P_{n}^{(\alpha,-1 / 2)}(\cos (\pi \theta / L))$. Here $\alpha=(d-2) / 2, d=2$, $3, \cdots$. For $P^{d}(C)$ the spherical functions are $P_{n}^{(\alpha, 0)}(\cos (\pi \theta / L))$, $\alpha=(d-2) / 2, d=4,6, \cdots$. For $P^{d}(H)$ they are $P_{n}^{(\alpha, 1)}(\cos (\pi \theta / L))$, $\alpha=(d-2) / 2, d=8,12, \cdots$, and for the Cayley elliptic plane they are $P_{n}^{(7,3)}(\cos (\pi \theta / L))$. See [4].

If each of these spaces has diameter equal to one we can isometrically imbed $P^{d}(R)$ in $P^{2 d}(C)$, which can be isometrically imbedded in $P^{4 d}(H)$. Also $P^{8}(H)$ can be isometrically imbedded in $P^{16}$ so we have that $\alpha_{k} \geqq 0$ for certain values of $\alpha, \beta, \gamma, \delta$. They are the values on the lines through $(-1,-1)$ of the form $(k / 2-1,-1 / 2),(k-1,0)$, $(2 k-1,1),(7,3), k=2,3, \cdots$.

In the other direction since $\alpha_{k}$ is not always greater than or equal to zero for points above these lines we have that you cannot isometrically imbed $P^{d+1}(R)$ in $P^{2 d}(C)$ or $P^{4 d}(H)$, that $P^{2 d+2}(C)$ cannot be isometrically imbedded in $P^{4 d}(H)$ and that $P^{3}(R), P^{6}(C)$ and $P^{12}(H)$ cannot be isometrically imbedded in $P^{16}$ when they have the same diameter. When the space with smaller real dimension has a larger diameter you clearly cannot imbed isometrically. If the diameter is smaller, then if you could isometrically imbed one of these spaces you could also isometrically imbed a circle of the same diameter. Thus we need to consider 


$$
P_{1}^{(\gamma, \delta)}\left(\cos \frac{\theta}{L}\right)=\sum_{k=0}^{\infty} \alpha_{k} \cos k \theta
$$

with $L>1$, and $\gamma>\delta \geqq-\frac{1}{2}$.

$$
P_{1}^{(\gamma, \delta)}(x)=((\gamma-\delta) / 2)+((\gamma+\delta+2) / 2) x
$$

and so

$$
\alpha_{k}=\frac{(\gamma+\delta+2)}{\pi} \int_{0}^{\pi} \cos \frac{\theta}{L} \cos k \theta d \theta, \quad k=1,2, \cdots
$$

A simple calculation shows that

$$
\alpha_{k}=(\gamma+\delta+2)(-1)^{k} \sin (\pi / L) / \pi L\left(k^{2}-1 / L^{2}\right)
$$

and since $L>1$ this is not always nonnegative.

\section{REFERENCES}

1. R. Askey and S. Wainger, A transplantation theorem for ultraspherical coefficients, Pacific J. Math. 16 (1966), 393-405.

2. S. Bochner, Hilbert distances and positive definite functions, Ann. of Math (2) 42 (1941), 647-656.

3. E. Feldheim, Contributions à la theorie des polynomes de Jacobi, Mat. Fiz. Lapok 48 (1941), 453-504. (Hungarian, French summary)

4. R. Gangolli, Positive definite kernels on homogeneous spaces and certain stochastic processes related to Levy's Brownian motion of several parameters, Ann. de l'Inst. Henri Poincaré, sect. B 3 (1967), 121-226.

5. L. Gegenbauer, Zur Theorie der Funktionen $C_{n}^{y}(x)$, Denksch. der Akad. der Wiss. in Wien, Math. natur. Klasse 48 (1884), 293-316.

6. L. K. Hua, Harmonic analysis of functions of several complex variables in the classical domains, Translation from the Russian, Amer. Math. Soc., Providence, R.I., 1963.

7. I. J. Schoenberg, Positive definite functions on spheres, Duke Math. J. 9 (1942), 96-108.

8. G. Szegö, Orthogonal polynomials, Amer. Math. Soc. Colloq. Publ., vol. 23, Amer. Math. Soc., Providence, R. I., 1959.

UNIVERSITY OF Wisconsin, MADISON 